\title{
Targeting Bacterial Sortase A with Covalent Inhibitors: 27 New Starting Points for Structure-Based Hit-to-Lead Optimization
}

Kristaps Jaudzems, Viktorija Kurbatska, Atis Jēkabsons, Raitis Bobrovs, Zhanna Rudevica, Ainars Leonchiks

\section{Contents}

Table S1 S2

Figure $\mathrm{S} 1$ S8

Figure $\mathrm{S} 2$ S9

Figure S3 $\mathrm{S} 10$

Figure S4 $\mathrm{S} 11$

Figure S5 $\mathrm{S} 12$

Figure S6 $\mathrm{S} 13$

Table S2 S14 
Table S1. Illustrative 2D ${ }^{15} \mathrm{~N}-{ }^{1} \mathrm{H}$ HSQC NMR spectra of ${ }^{15} \mathrm{~N}$ labeled $\mathrm{Sa}$-SrtA showing the covalent binding of inhibitors (one per each inhibitor class, except the unclassified inhibitors, for which all the spectra are shown). The spectra shown are in absence of inhibitor (labeled "no inhibitor"), after addition of $0.5,1,2$ or 4 equivalents of inhibitor (labeled " $0.5 \mathrm{x}$ ", " $1 \mathrm{x}$ ", " $2 \mathrm{x}$ " and " $4 \mathrm{x}$ ") and after dialysis against NMR buffer (labeled "dialyzed").

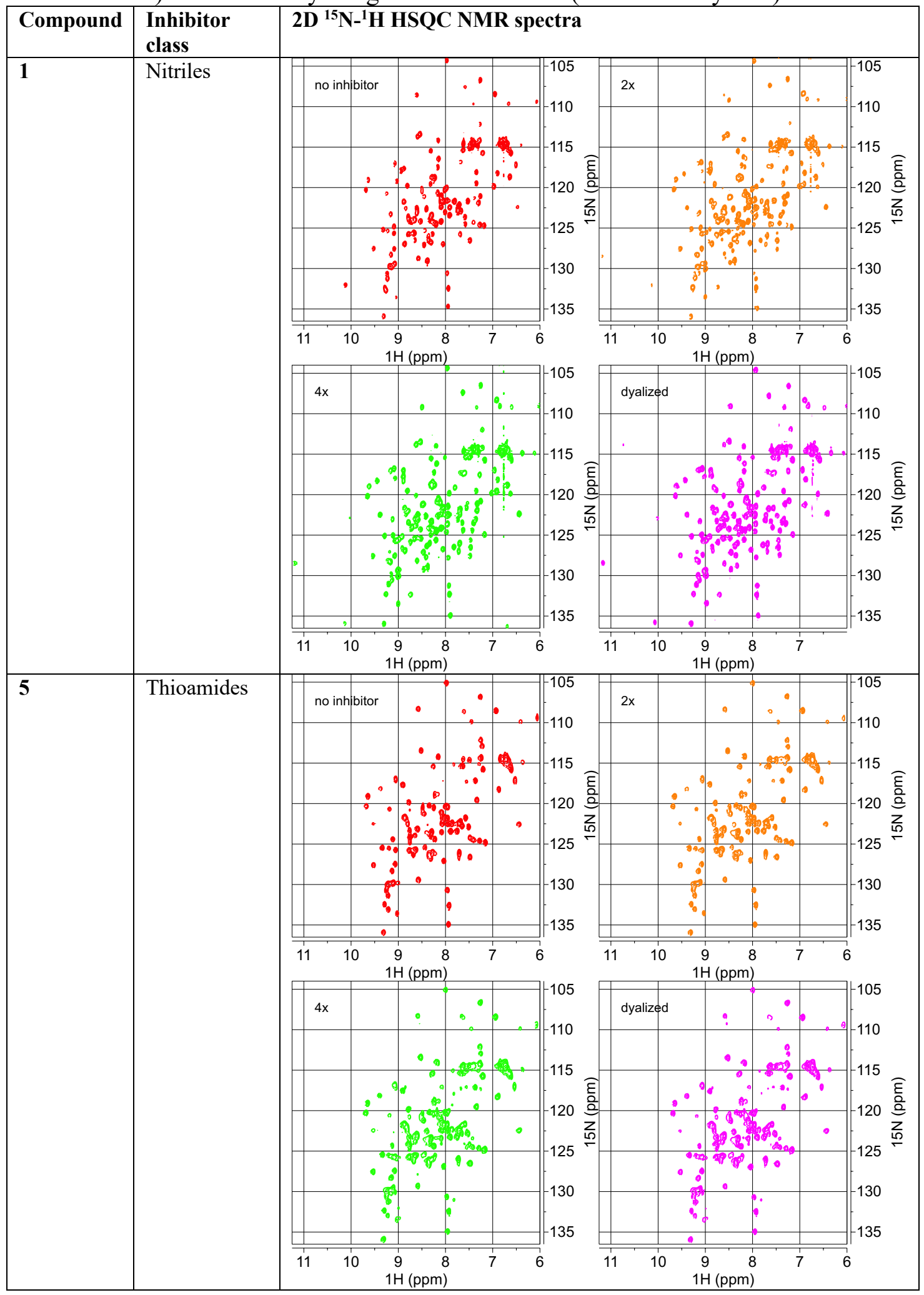




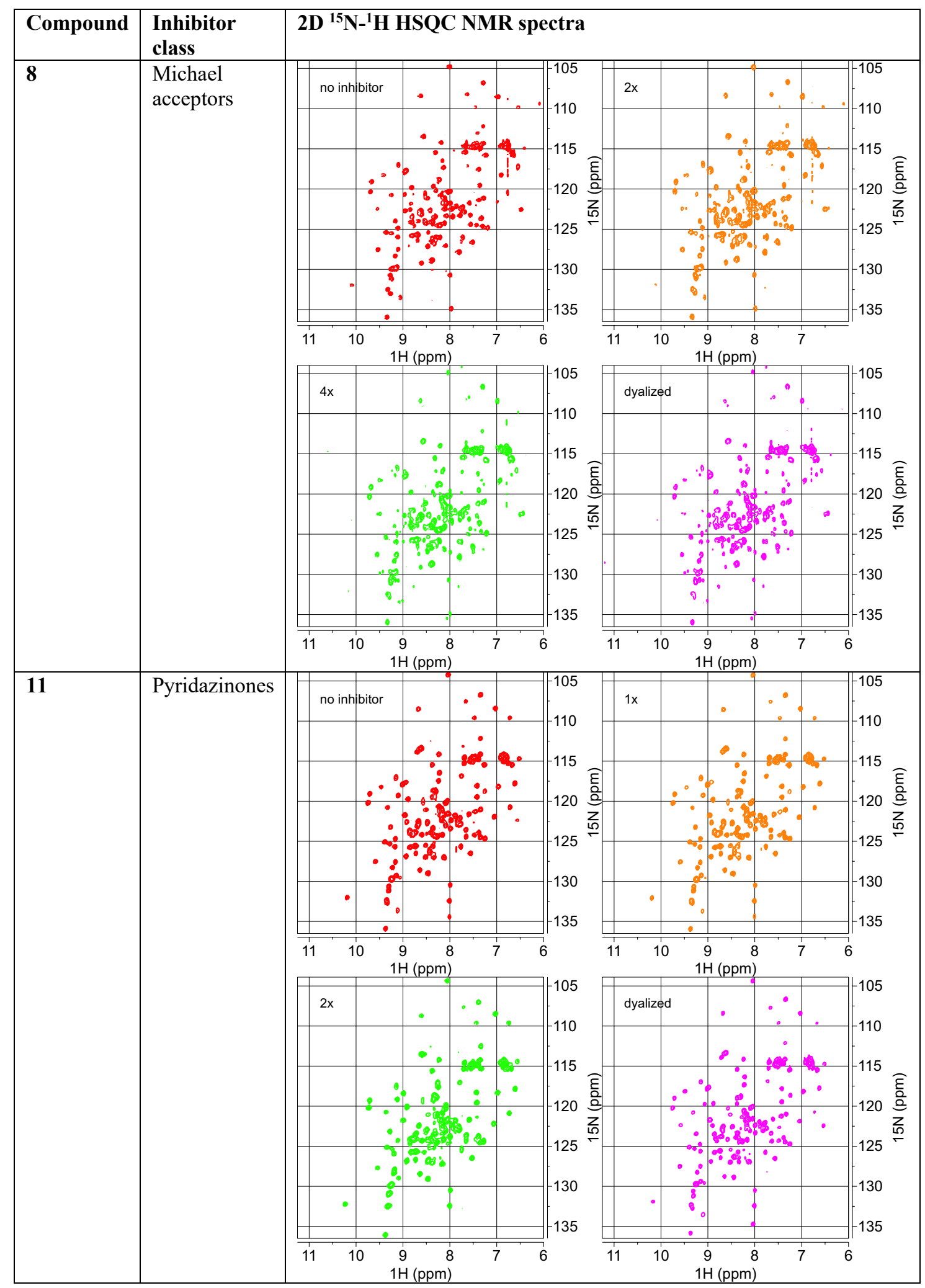




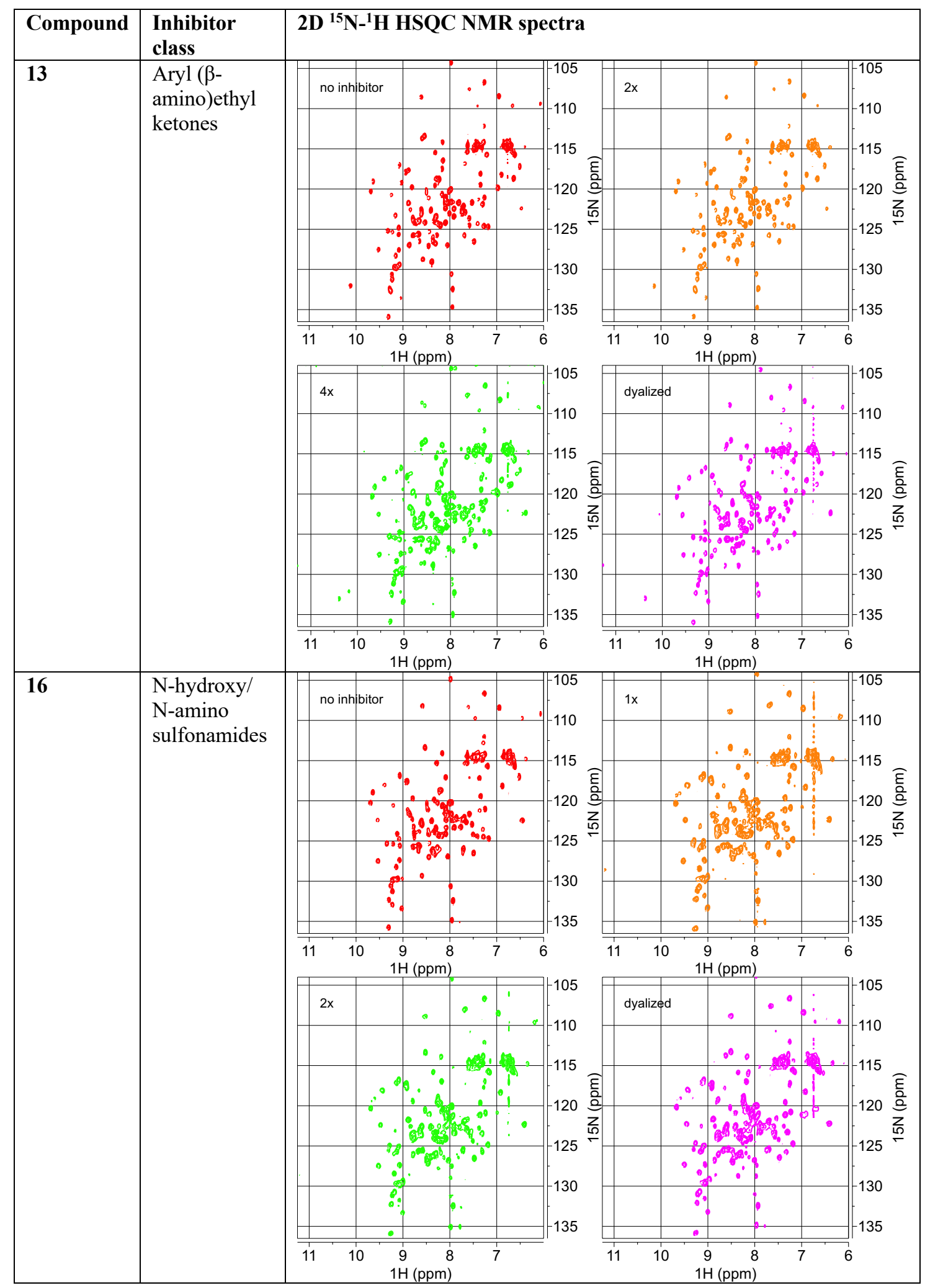




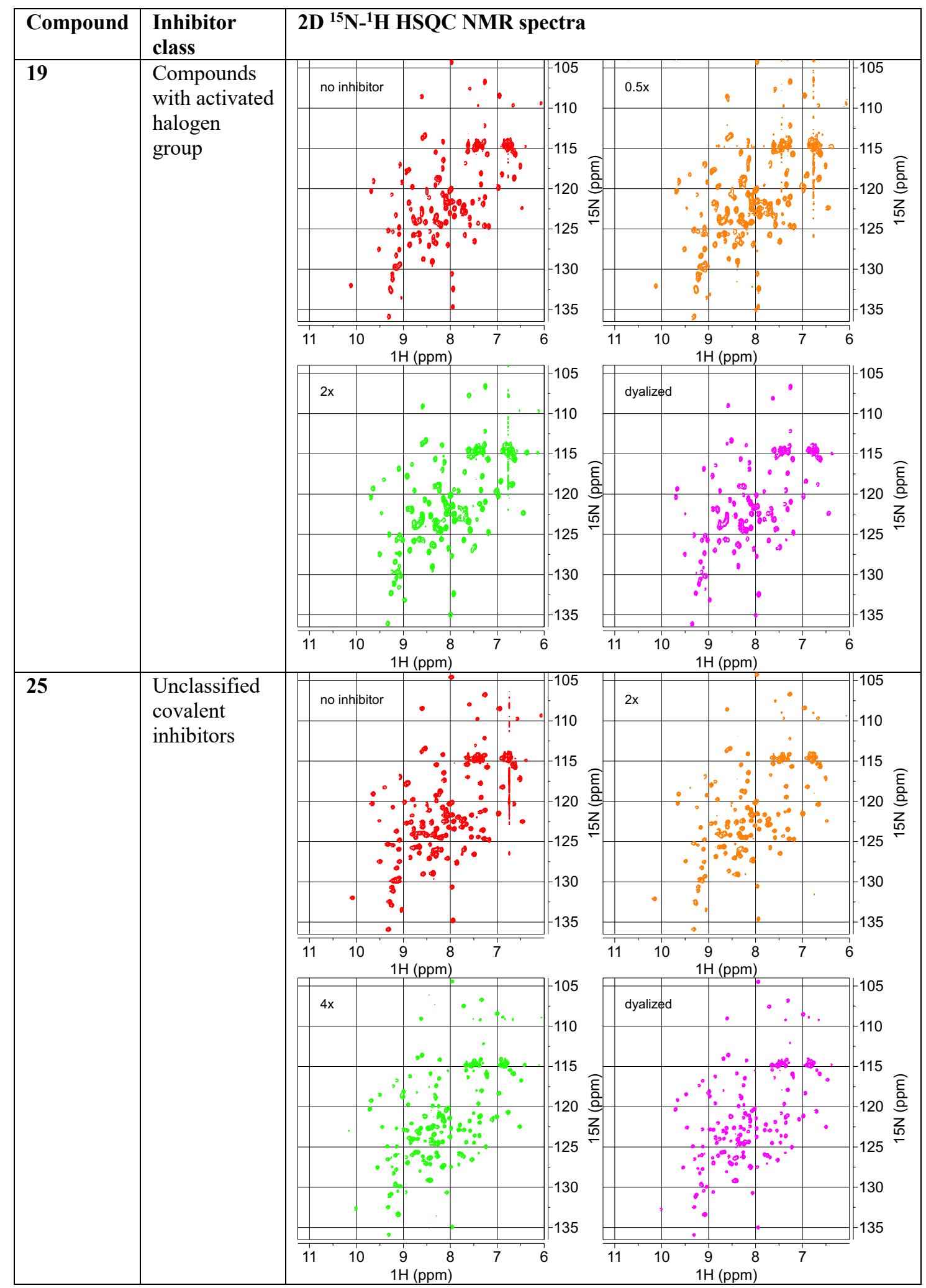




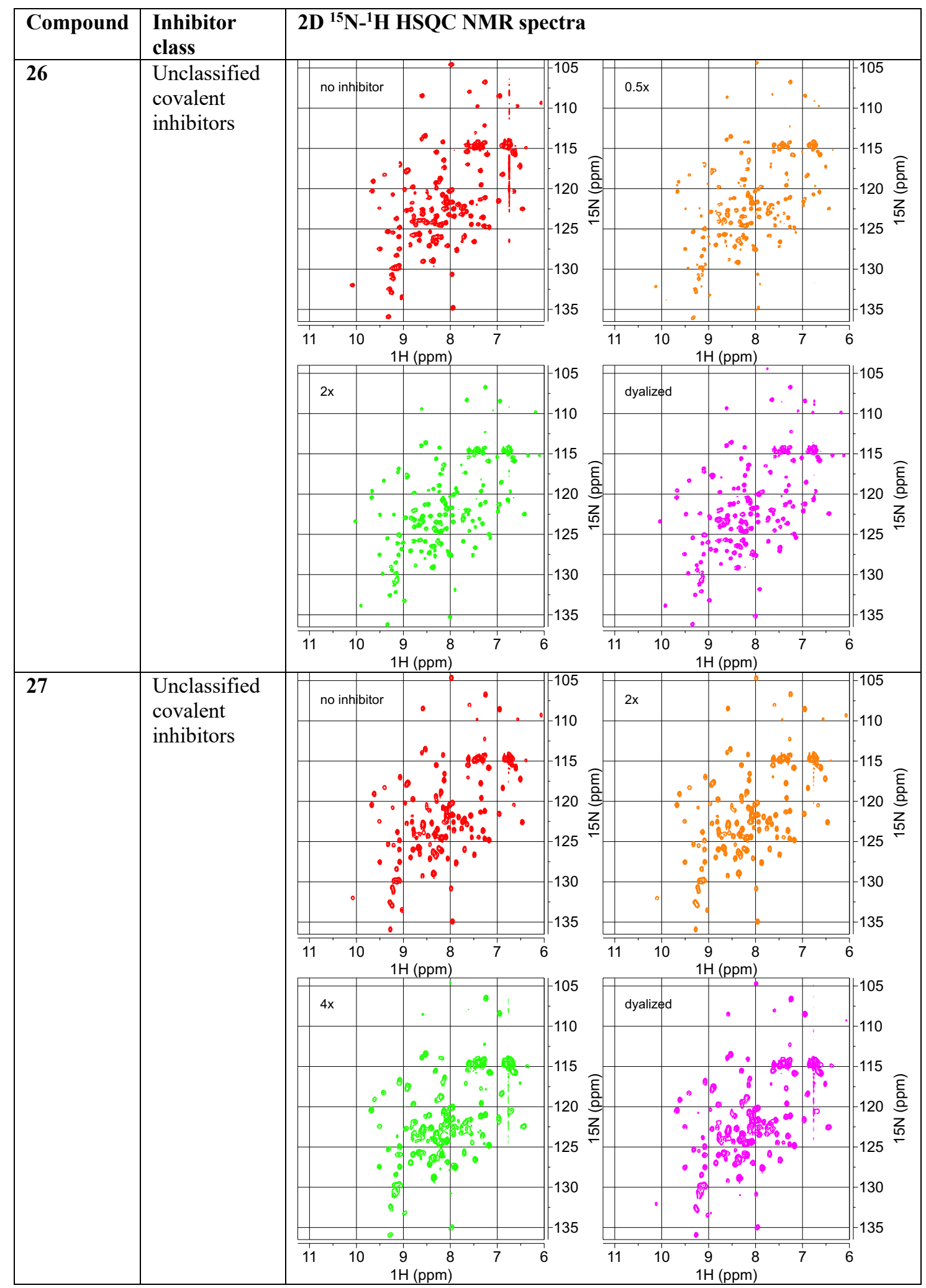




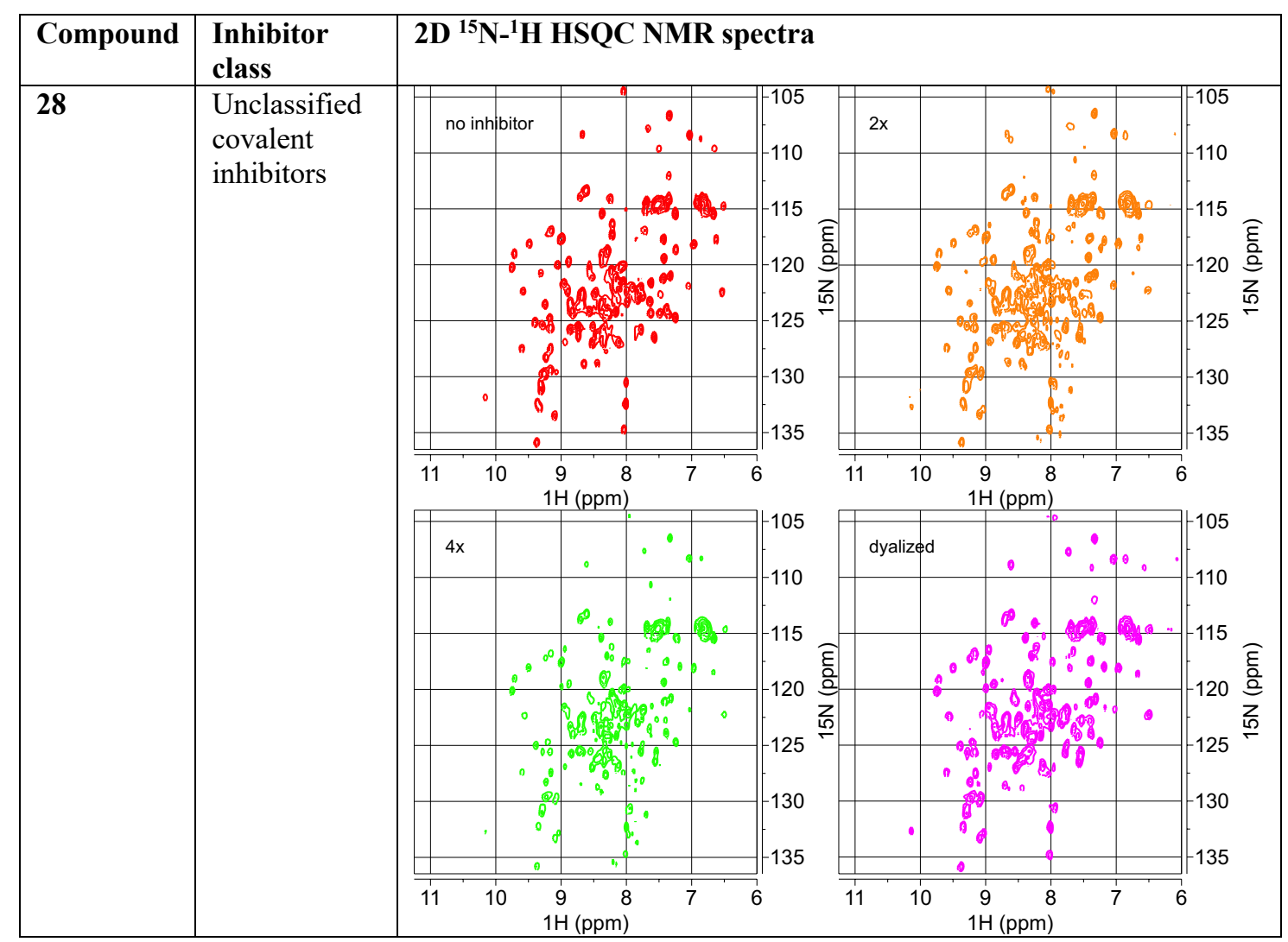



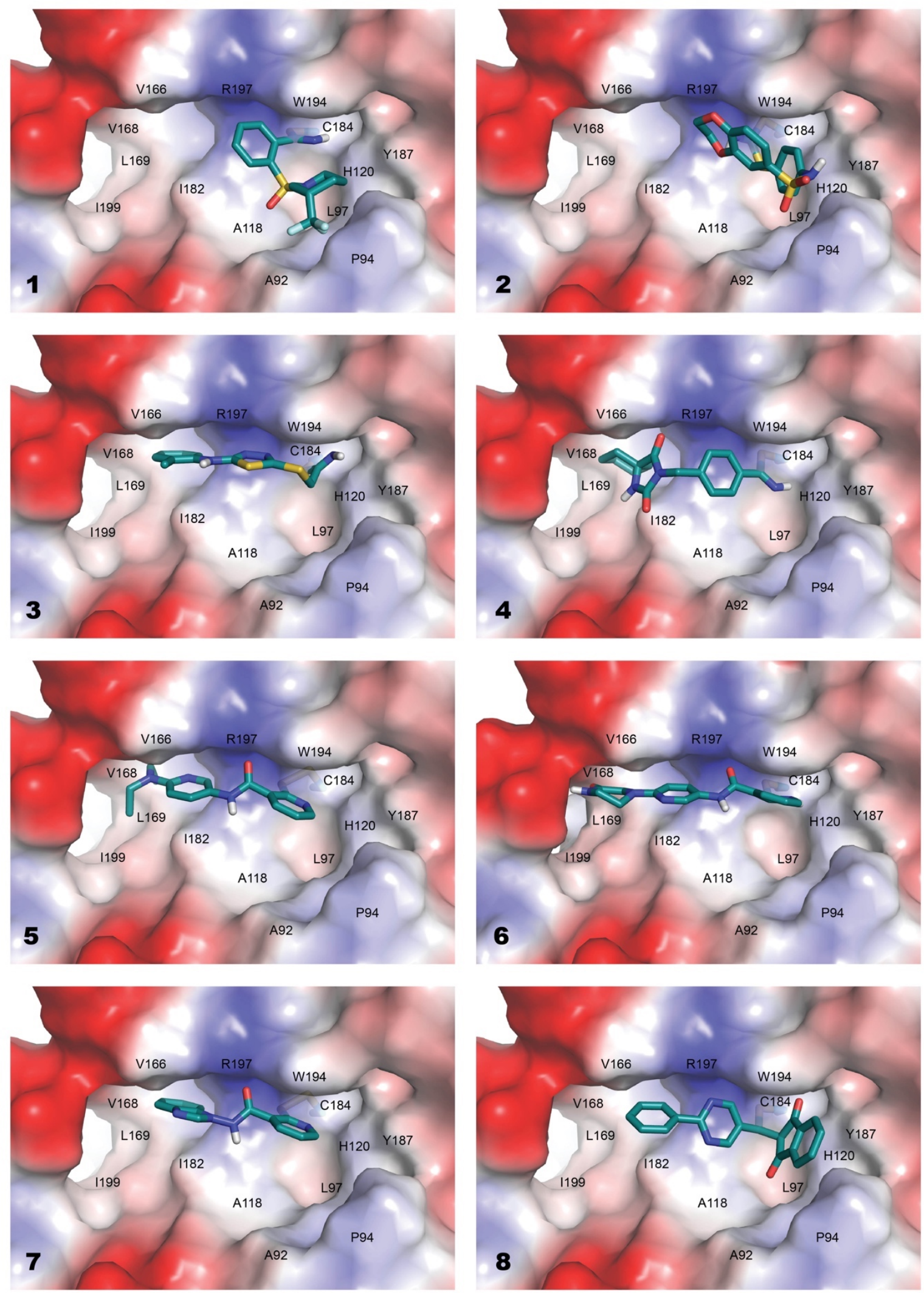

Figure S1. Covalent docking models of inhibitors 1-8. The electrostatic potential surface of the $\mathrm{Sa}$-SrtA active site cavity is shown and locations of selected amino acid residues are indicated, the inhibitors are shown with sticks. Negatively charged areas are colored red and positively charged areas are blue. Prepared using The PyMOL Molecular Graphics System, version 2.1.0 Schrödinger, LLC. 

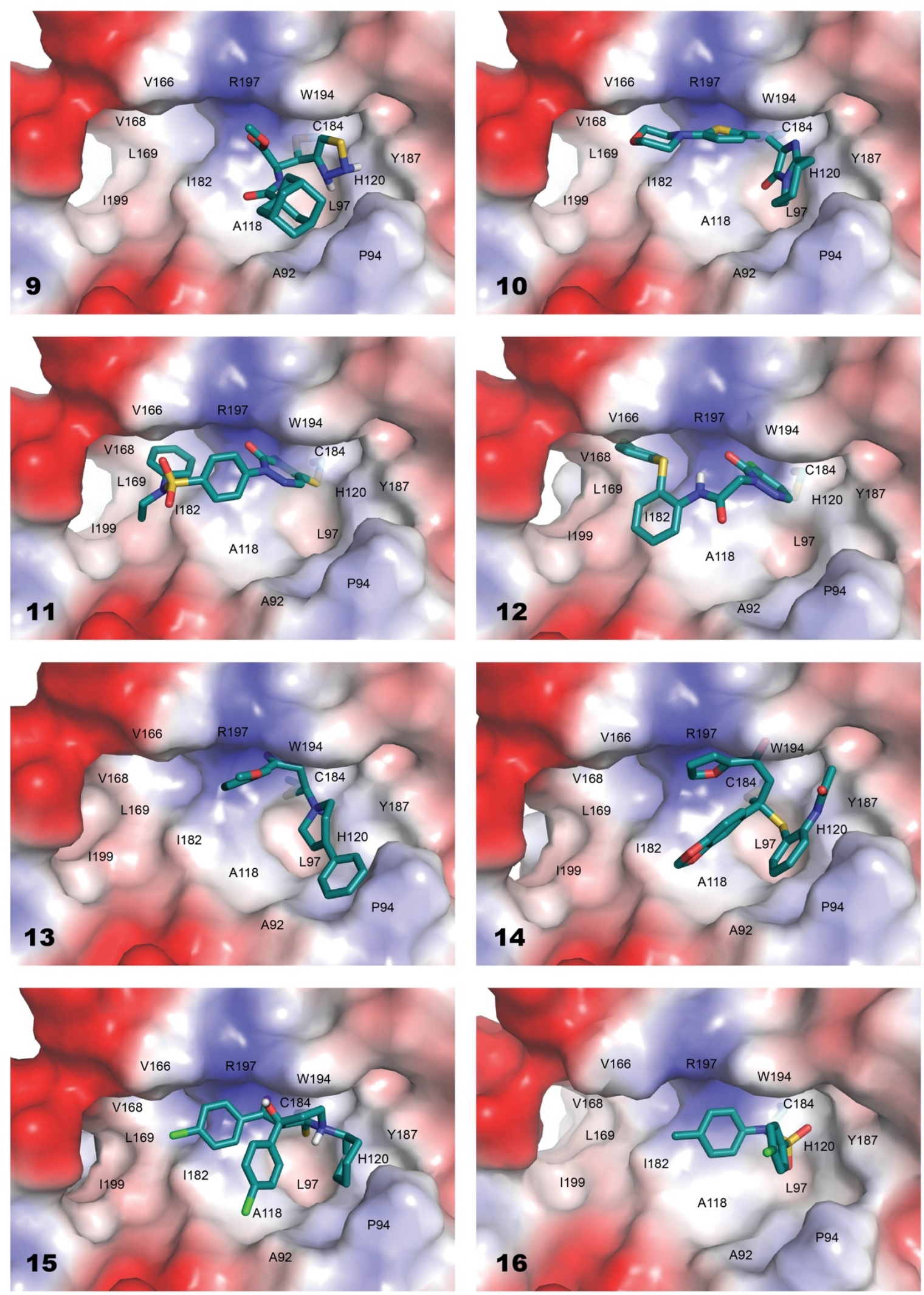

Figure S2. Covalent docking models of inhibitors 9-16. The electrostatic potential surface of the $\mathrm{Sa}$-SrtA active site cavity is shown and locations of selected amino acid residues are indicated, the inhibitors are shown with sticks. Negatively charged areas are colored red and positively charged areas are blue. Prepared using The PyMOL Molecular Graphics System, version 2.1.0 Schrödinger, LLC. 

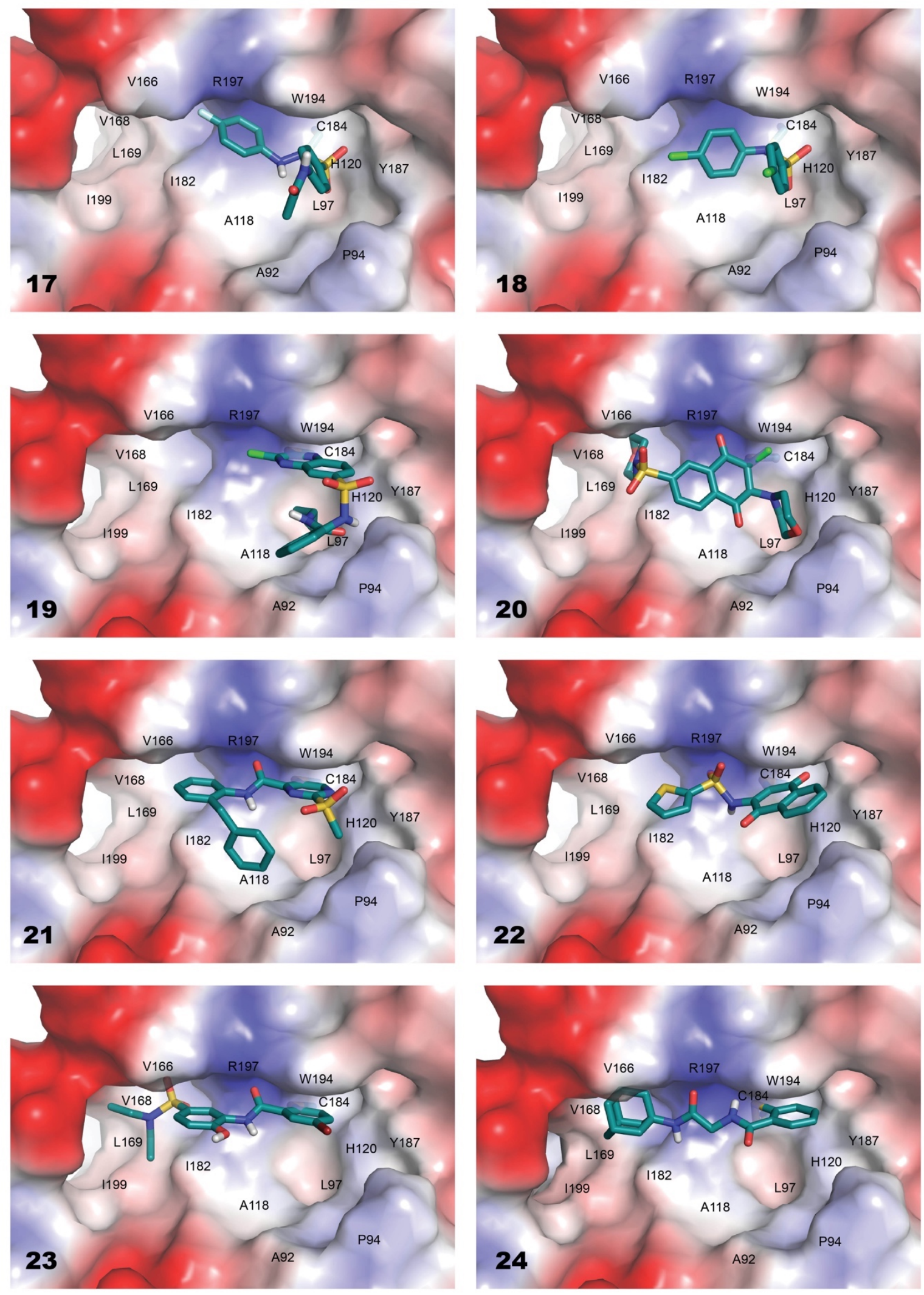

Figure S3. Covalent docking models of inhibitors 17-24. The electrostatic potential surface of the $\mathrm{Sa}$-SrtA active site cavity is shown and locations of selected amino acid residues are indicated, the inhibitors are shown with sticks. Negatively charged areas are colored red and positively charged areas are blue. Prepared using The PyMOL Molecular Graphics System, version 2.1.0 Schrödinger, LLC. 


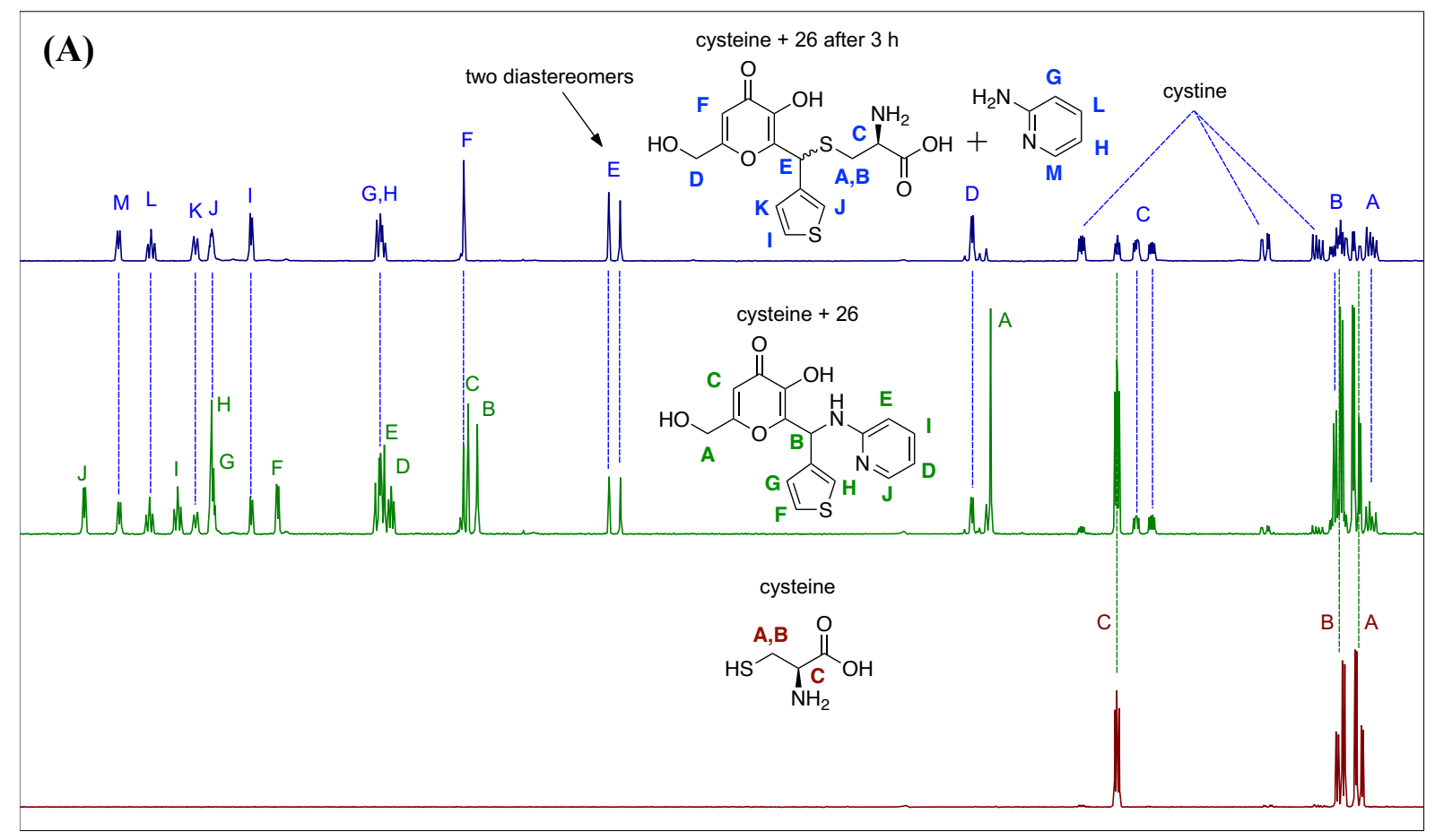

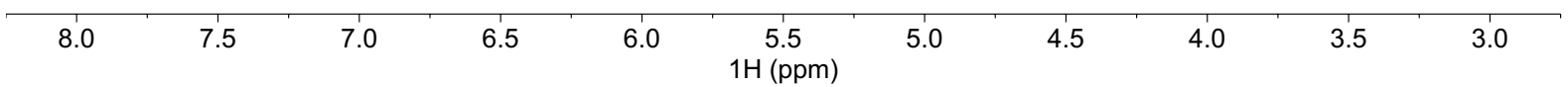

(B)

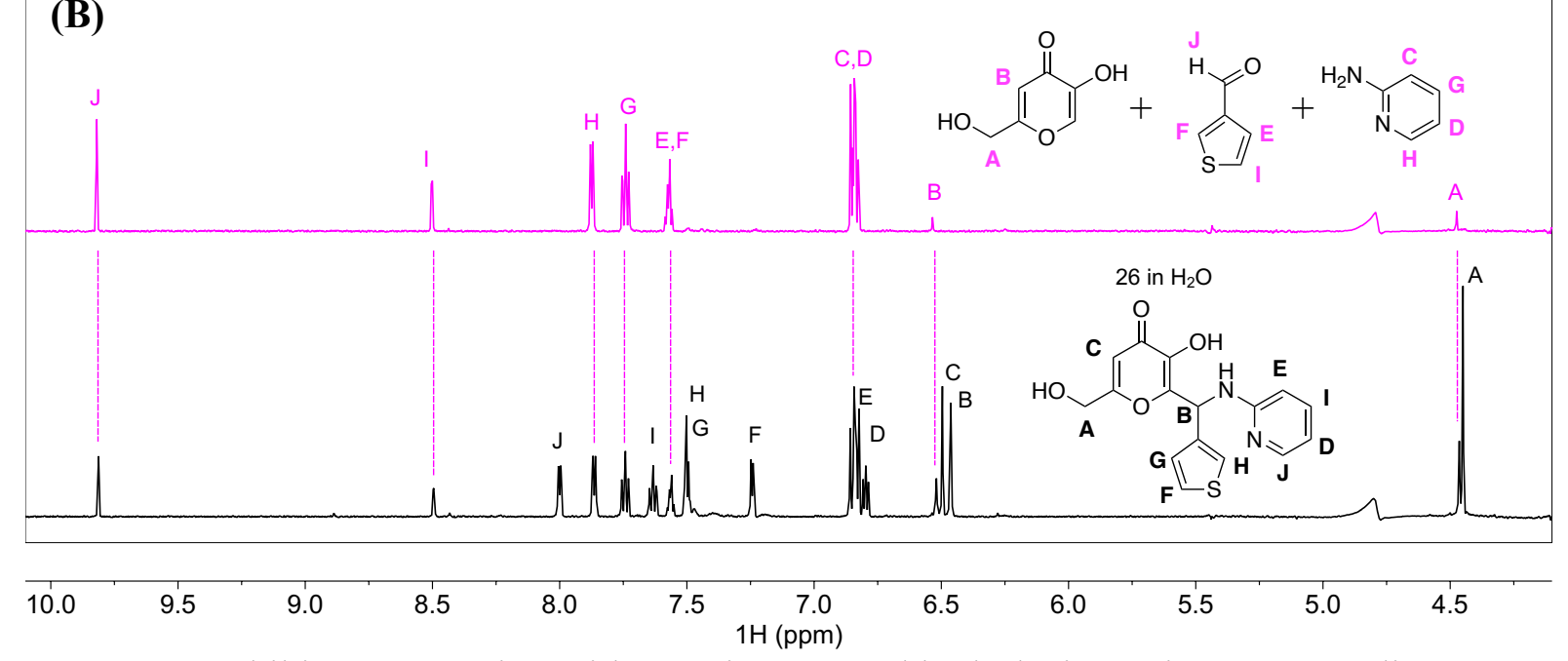

Figure S4. Inhibitor 26 reaction with cysteine (A) and hydrolysis (B) in $20 \mathrm{mM}$ sodium phosphate buffer, $\mathrm{pH} 7.8,5 \% \mathrm{D}_{2} \mathrm{O}, 7.1 \%$ DMSO-d 6 monitored by ${ }^{1} \mathrm{H}$ NMR. 


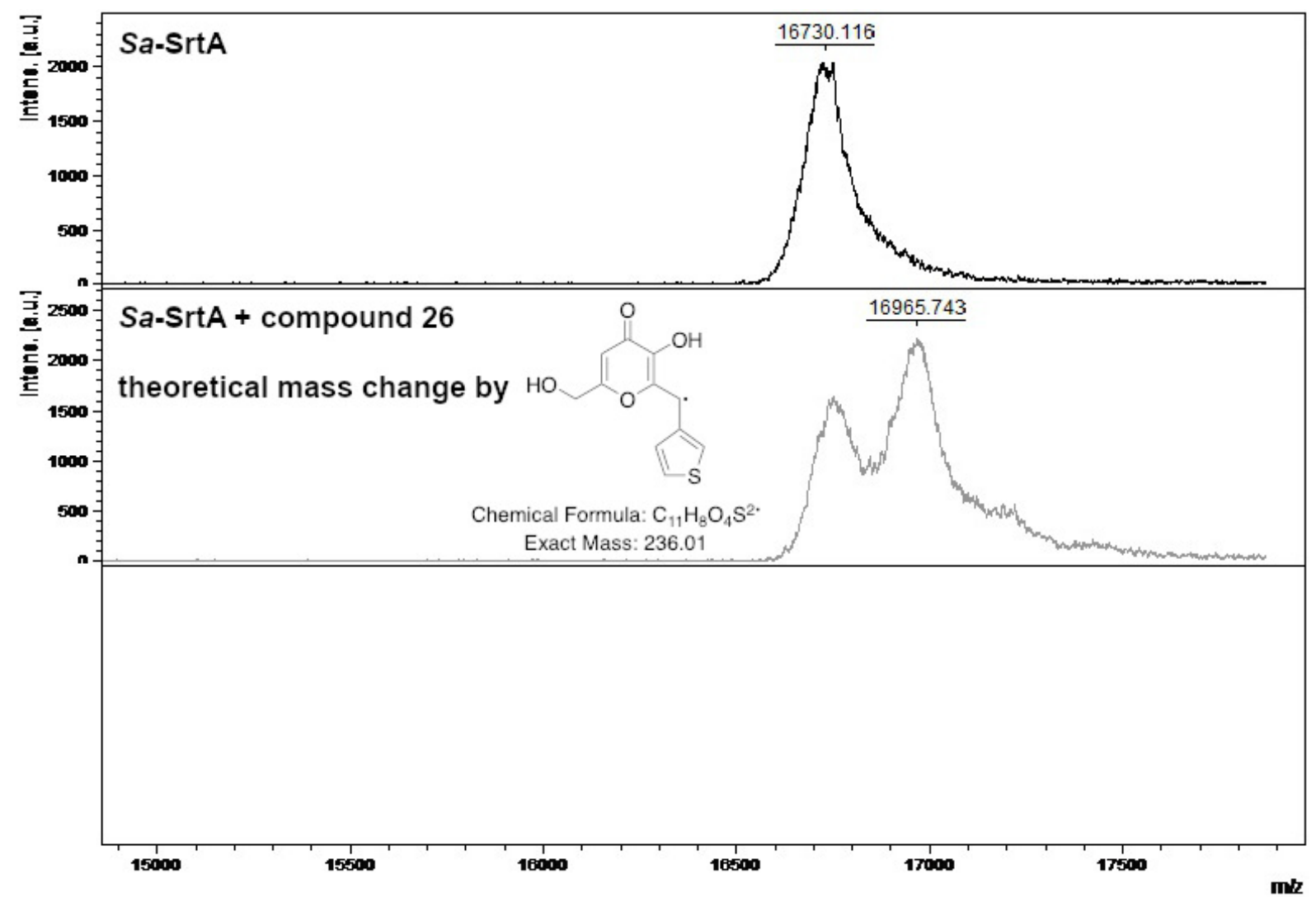

Figure S5. MALDI-TOF-MS analysis of $\mathrm{Sa}$-SrtA and compound $\mathbf{2 6}$ adduct formation. 10 $\mu \mathrm{M} S a$-SrtA stock solution was mixed with $500 \mu \mathrm{M}$ compound 26 in $100 \mu 1$ reaction buffer (50 mM Tris-HCl, $150 \mathrm{mM} \mathrm{NaCl}, \mathrm{pH} 7.5$ ) and incubated for $30 \mathrm{~min}$. at room temperature. The protein-compound samples and protein calibration standards were prepared according to the dried droplet method by mixing $2 \mu \mathrm{L}$ sample, $2 \mu \mathrm{L} 0.1 \%$ trifluoro acetic acid and $2 \mu \mathrm{L}$ DHAP matrix solutions. Afterwards, $1 \mu \mathrm{L}$ of the above mix was applied onto a standard steel target and dried at room temperature for 1 hour. The samples were tested using Bruker matrix-assisted laser desorption and ionization time-of-flight mass spectrometer (MALDITOF-MS) and spectra were analyzed by FlexAnalysis software. 
Compound 26
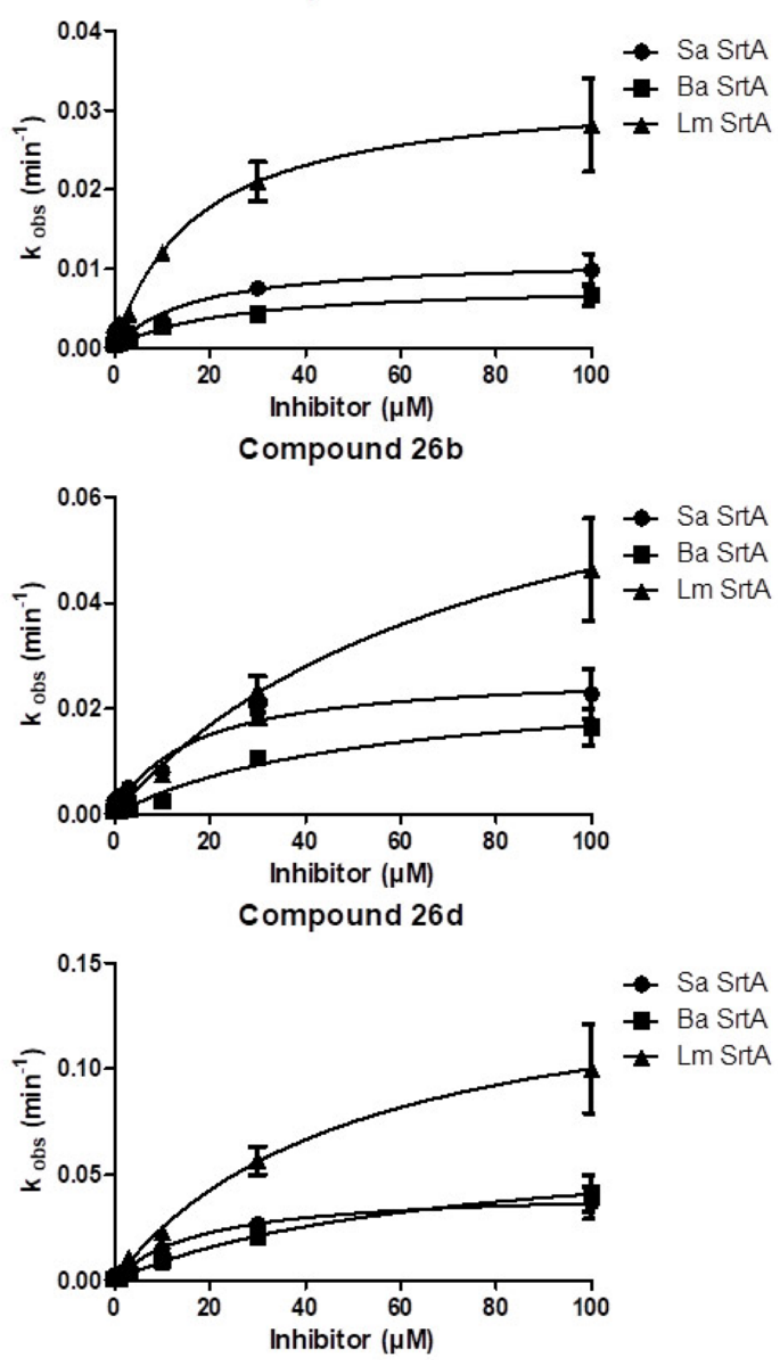

Compound 26a

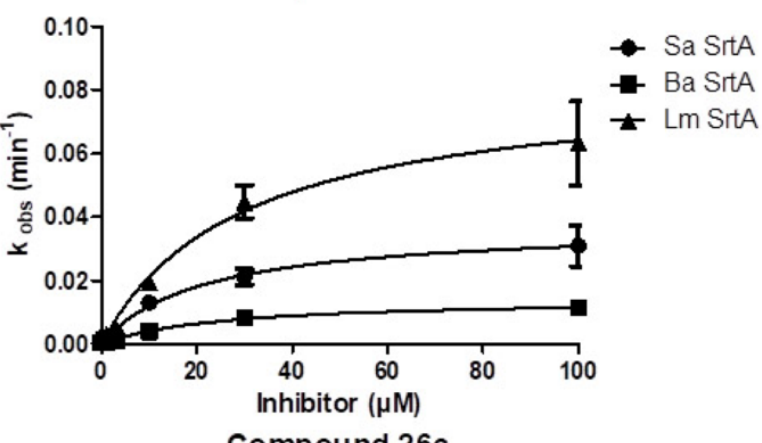

Compound 26c
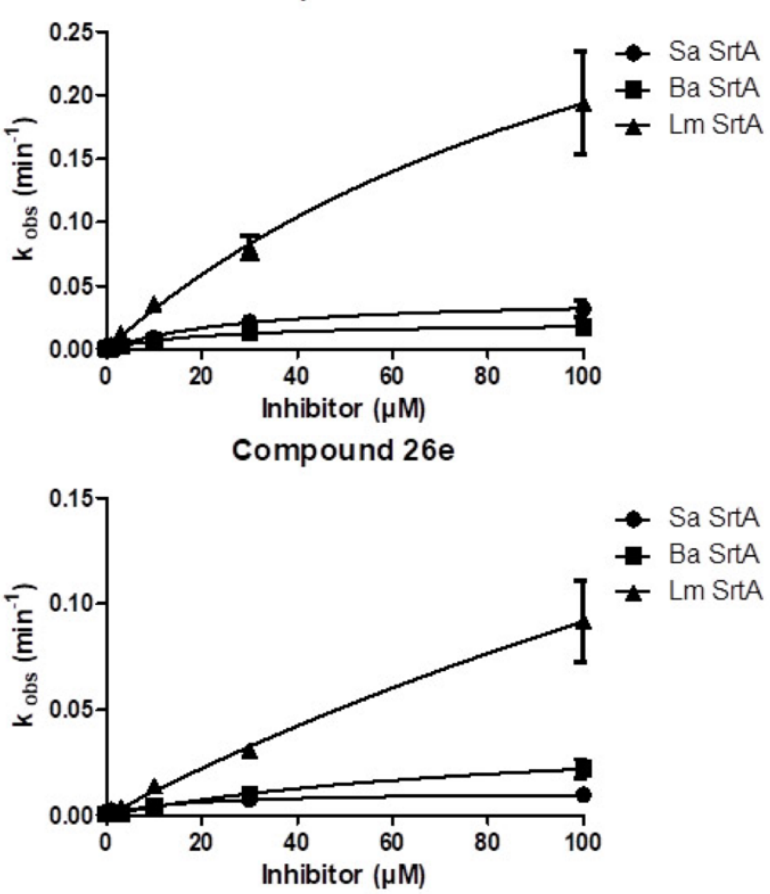

Figure S6. $\mathrm{Sa}$-SrtA, $\mathrm{Lm}$-SrtA and $\mathrm{Ba}$-SrtA inactivation kinetics. The rate of SrtA inhibition by compounds 26, 26a, 26b, 26c, 26d and 26e was determined by calculating $k_{\text {inact }} / K_{\text {I,app }}$ as described in Methods. 
Table S2. Input for structure calculation and structural statistics of the NMR structure of inhibitor 26-Sa-SrtA complex.

\begin{tabular}{|c|c|}
\hline Quantity & Value $^{\mathrm{a}}$ \\
\hline NOE upper distance limits & 2265 \\
\hline intra-residual $(|\mathrm{i}-\mathrm{j}|=0)$ & 628 \\
\hline short-range & 641 \\
\hline medium-range & 310 \\
\hline long-range & 686 \\
\hline inhibitor-to-protein & 9 \\
\hline H-bond restraints & 40 \\
\hline \multicolumn{2}{|l|}{ Residual NOE violations } \\
\hline number $\geq 0.1 \AA$ & $34 \pm 4$ \\
\hline $\operatorname{maximum}[\AA]$ & $0.42 \pm 0.18$ \\
\hline \multicolumn{2}{|l|}{ PARALLHDG energies [kcal/mol] } \\
\hline total & $-5765 \pm 108$ \\
\hline van der Waals & $-650 \pm 25$ \\
\hline electrostatic & $-6298 \pm 122$ \\
\hline \multicolumn{2}{|l|}{ R.m.s.d. from mean coordinates $[\AA]$} \\
\hline backbone (residues $63-119,130-158,176-183,198-206)$ & $0.56 \pm 0.05$ \\
\hline all heavy atoms (residues $63-119,130-158,176-183,198-206$ ) & $0.97 \pm 0.06$ \\
\hline all heavy atoms (inhibitor 26) & $1.48 \pm 0.60$ \\
\hline \multicolumn{2}{|l|}{ Ramachandran plot statistics } \\
\hline most favored regions [\%] & 76.9 \\
\hline additional allowed regions [\%] & 22.0 \\
\hline generously allowed regions [\%] & 0.9 \\
\hline disallowed regions [\%] & 0.2 \\
\hline
\end{tabular}

${ }^{a}$ Except for the top six entries, average values and standard deviations for the 20 energyminimized conformers are given. 